\title{
Transporte interhospitalario pediátrico: Una necesidad creciente de la red asistencial chilena
}

\author{
MARIE JESIE CARRILLO B. ${ }^{1}$ \\ 1. Enfermera Matrona. Docente. Pontificia Universidad Católica de Chile.
}

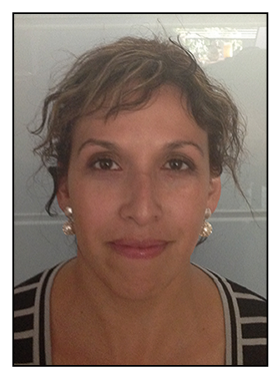

El Ministerio de Salud Chileno, por medio de la Subsecretaria de Redes Asistenciales, articula y coordina la red con el objetivo de permitir el acceso del paciente a los recursos médicos y tecnológicos que requiere, por lo cual la necesidad de transporte interhospitalario es una actividad creciente. Esta actividad de transporte es efectuada principalmente a través del Servicio de Atención Médica de Urgencia (SAMU), que realiza el transporte de los pacientes según sus requerimientos de salud, siendo prioritario y de vital importancia en el paciente en estado crítico ${ }^{1}$.

El SAMU realiza transporte tanto de pacientes adultos como pediátricos, siendo los primeros en mayor número y los segundos con requerimientos extremadamente especializados, sobretodo dados los avances en cuidados críticos a nivel infantil, lo que ha generado una creciente necesidad de transporte de niños críticamente enfermos a instituciones especializadas. Esta situación que se ve reflejada en el aumento del transporte de pacientes pediátricos menores de 2 años durante el año 2013 por esta institución ${ }^{2}$.

El creciente desarrollo tecnológico de soporte vital y el aumento de niños con necesidades especiales en salud, ha acrecentado la necesidad de transporte pediátrico a unidades de cuidados críticos (UCIP) ${ }^{3,4}$.
Un aspecto importante del transporte pediátrico es el reconocimiento oportuno de los pacientes con riesgo de complicaciones, en donde el paro cardiorrespiratorio (PCR) reviste mayor gravedad, complicación que presenta una tasa publicada entre 0,1 a 20 por 1.000 niños ingresados en unidades de hospitalización. Adicionalmente, esta complicación se asocia con baja supervivencia y morbilidad significativa en los sobrevivientes ${ }^{5-7}$.

La $5^{\circ}$ Conferencia en transporte pediátrico y neonatal realizada por la División de Transporte Médico de la Academia Americana de Pediatría, concluyó la necesidad de formación de protocolos y/o recomendaciones para mejorar las prácticas y la toma de decisión del transporte pediátrico ${ }^{3,4}$, lo que muestra la necesidad de investigar en esta área.

Las investigaciones publicadas relacionadas con transporte pediátrico se han centrado principalmente en la composición óptima del equipo que realiza el traslado, mostrando la mayoría de los reportes una amplia diversidad en las prácticas clínicas. Otras investigaciones se han enfocado en la evaluación de la gravedad del paciente y en los sistemas de puntuación o categorización necesarios para un sistema de transporte de pacientes pediátricos ${ }^{8,9}$.

De estas últimas investigaciones se infiere adicionalmente que el transporte de pacientes

Recibido el 13 de marzo de 2014, devuelto para corregir el 17 de marzo de 2014, segunda versión 01 de abril de 2014, aceptado para publicación el 08 de abril de 2014.

Correspondencia a:

Marie Jesie Carrillo Barrera

E-mail: mcarrilb@uc.cl 
pediátricos requiere de protocolos para determinar la necesidad del transporte y la exposición al deterioro fisiopatológico que podría sufrir el paciente durante el mismo.

\section{Evaluación de la necesidad de transporte}

La necesidad de poder determinar que pacientes pediátricos presentan riesgo de deterioro dentro del hospital e identificar el transporte oportuno a una unidad de cuidados intensivos Pediatrica (UCIP) ha generado la creación de algunos Puntajes de Gravedad, en donde el denominado "Sistema Pediátrico de Alerta Temprana" (PEWS) presenta mayor sensibilidad y especificidad. Es un sistema de puntuación de 20 variables desarrollado en primera instancia por el equipo del Dr. Duncan en Canadá. Este equipo realizo un estudio de casos y controles con un total de 128 controles y 87 casos. Los pacientes "casos" presentaron riesgo de Paro cardiorrespiratorio (PCR) o definitivamente PCR y se trasladaron a UCIP, los "controles" fueron los pacientes pediátricos que no tuvieron riesgo de PCR y no tuvieron necesidad de ingreso a UCIP. La investigación demostró que el PEWS es una excelente herramienta diagnostica para predecir PCR, con un ROC de 0,9 , un $78 \%$ de sensibilidad y un $95 \%$ de especificidad para un score de 5 puntos y más ${ }^{10}$.

Posteriormente, el score PEWS fue perfeccionado durante el año 2009 por el grupo de Parshuram y cols, utilizando un estudio de cohorte, con 60 pacientes pediátricos hospitalizados en UCIP y otra de 120 pacientes pediátricos hospitalizados en sala no UCIP, se utilizaron sólo 7 ítems del PEWS inicial: frecuencia cardíaca, presión arterial sistólica, tiempo de llenado capilar, frecuencia respiratoria, esfuerzo respiratorio, saturación de oxígeno y terapia de oxígeno. Cada ítem, presento una puntuación de 0 a 4, según valores ajustado a grupos de edades (tabla 1). Finalmente se concluyó que el PEWS de 7 ítems, obtuvo un ROC 0,91 para una puntuación sobre 7 y además se pudo identificar en un $80 \%$ los pacientes pediátricos con riesgo de realizar un PCR antes de 1 hora. Demostrándose que el PEWS es una buena herramienta como evaluación de gravedad y riesgo de deterioro fisiológico. El estudio también concluye que puntuaciones sobre 5 requieren cama UCIP ${ }^{11}$. Según lo antes descrito el uso del PEWS como herramienta para evaluar y determinar la necesidad de transporte a UCIP, podría resultar de utilidad, quedando pendiente investigaciones nacionales que permitan validar su uso.

\section{Evaluación del deterioro fisiopatológico del paciente pediátrico durante el transporte}

Variadas publicaciones refieren que el transponte de un paciente crítico genera deterioro fisiopatológico con riesgo de eventos adversos como PCR ${ }^{12}$.

Este deterioro depende de algunos de los siguientes aspectos; impacto fisiológico del transporte, la patología aguda del paciente y la ocurrencia de eventos adversos durante el traslado.

En relación al impacto fisiológico del transporte, según describen variadas publicaciones, esto se produce por dos mecanismos principales; el desencadenado por los movimientos del paciente durante el traslado: aceleración y desaceleración, cambios posturales y el movimiento de una superficie a otra ${ }^{13-16}$ y el desencadenado por el cambio del entorno de protección de la unidad de cuidado inicial ${ }^{17,18}$.

En relación a la patología aguda que cursa el paciente pediátrico, una revisión sistemática realizada por Thompson y cols, refiere que los síntomas como tos, dolor abdominal, vómitos, diarrea, falta de apetito y coriza, resultan ser de menor gravedad en los pacientes pediátricos y con menos riesgo de complicaciones durante el transporte ${ }^{18}$. Lo descrito concuerda con otra investigación retrospectiva, realizada en Noruega, con 761 pacientes pediátricos, donde las enfermedades de vías áreas superiores y patologías gastrointestinal se asociaron con un PEWS bajo, a diferencia de los pacientes con enfermedades respiratorias inferiores y/o enfermedad cardiovascular se asociaron a un PEWS mayor, con necesidad de estabilización y transporte a UCIP ${ }^{19,20}$.

El último aspecto a considerar durante el transporte de pacientes críticos, son los eventos adversos. Fanara y cols publicaron, durante el año 2010, una revisión de 66 artículos que 
Tabla 1. Sistema Pediátrico de Alerta Temprana (PEWS)

\begin{tabular}{|c|c|c|c|c|c|}
\hline \multirow[t]{2}{*}{ Ítem } & \multirow[t]{2}{*}{ Grupo de edad } & \multicolumn{4}{|c|}{ Puntaje de ítem } \\
\hline & & 0 & 1 & 2 & 4 \\
\hline $\begin{array}{l}\text { Frecuencia cardíaca } \\
(\text { lpm) }\end{array}$ & $\begin{array}{l}0 \text { a }<3 \text { meses } \\
3 \text { a }<12 \text { meses } \\
1 \text { a } 4 \text { años } \\
>4 \text { a } 12 \text { años } \\
>12 \text { años }\end{array}$ & $\begin{array}{r}>110 y<150 \\
>100 y<150 \\
>90 y<120 \\
>70 y<110 \\
>60 y<100\end{array}$ & $\begin{array}{l}\geq 150 \text { ó } \leq 110 \\
\geq 150 \text { ó } \leq 100 \\
\geq 120 \text { ó } \leq 90 \\
\geq 110 \text { ó } \leq 70 \\
\geq 100 \text { ó } \leq 60\end{array}$ & $\begin{array}{l}\geq 180 \text { ó } \leq 90 \\
\geq 170 \text { ó } \leq 80 \\
\geq 150 \text { ó } \leq 70 \\
\geq 130 \text { ó } \leq 60 \\
\geq 120 \text { ó } \leq 50\end{array}$ & $\begin{array}{l}\geq 190 \text { ó } \leq 80 \\
\geq 180 \text { ó } \leq 70 \\
\geq 170 \text { ó } \leq 60 \\
\geq 150 \text { ó } \leq 50 \\
\geq 140 \text { ó } \leq 40\end{array}$ \\
\hline $\begin{array}{l}\text { Presión arterial } \\
\text { sistólica (mmHg) }\end{array}$ & $\begin{array}{l}0 \text { a }<3 \text { meses } \\
3 \text { a }<12 \text { meses } \\
1 \text { a } 4 \text { años } \\
>4 \text { a } 12 \text { años } \\
>12 \text { años }\end{array}$ & $\begin{array}{r}>60 y<80 \\
>80 y<100 \\
>90 y<110 \\
>90 y<120 \\
>100 y<130\end{array}$ & $\begin{array}{r}\geq 80 \text { ó } \leq 60 \\
\geq 100 \text { ó } \leq 80 \\
\geq 110 \text { ó } \leq 90 \\
\geq 120 \text { ó } \leq 90 \\
\geq 130 \text { ó } \leq 100\end{array}$ & $\begin{array}{l}\geq 100 \text { ó } \leq 50 \\
\geq 120 \text { ó } \leq 70 \\
\geq 125 \text { ó } \leq 75 \\
\geq 140 \text { ó } \leq 80 \\
\geq 150 \text { ó } \leq 85\end{array}$ & $\begin{array}{l}\geq 130 \text { ó } \leq 45 \\
\geq 150 \text { ó } \leq 60 \\
\geq 160 \text { ó } \leq 65 \\
\geq 170 \text { ó } \leq 70 \\
\geq 190 \text { ó } \leq 75\end{array}$ \\
\hline $\begin{array}{l}\text { Tiempo de llenado } \\
\text { capilar }\end{array}$ & & $<3$ segundos & & & $\geq 3$ segundos \\
\hline $\begin{array}{l}\text { Frecuencia respira- } \\
\text { toria (respiraciones } \\
\text { por min) }\end{array}$ & $\begin{array}{l}0 \text { a }<3 \text { meses } \\
3 \text { a }<12 \text { meses } \\
1 \text { a } 4 \text { años } \\
>4 \text { a } 12 \text { años } \\
>12 \text { años }\end{array}$ & $\begin{array}{l}>29 y<61 \\
>24 \text { ó }<51 \\
>19 \text { ó }<41 \\
>19 \text { ó }<31 \\
>11 \text { ó }<17\end{array}$ & $\begin{array}{l}\geq 61 \text { ó } \leq 29 \\
\geq 51 \text { ó } \leq 24 \\
\geq 41 \text { ó } \leq 19 \\
\geq 31 \text { ó } \leq 19 \\
\geq 17 \text { ó } \leq 11\end{array}$ & $\begin{array}{l}\geq 81 \text { ó } \leq 19 \\
\geq 71 \text { ó } \leq 19 \\
\geq 61 \text { ó } \leq 15 \\
\geq 41 \text { ó } \leq 14 \\
\geq 23 \text { ó } \leq 10\end{array}$ & $\begin{array}{l}\geq 91 \text { ó } \leq 15 \\
\geq 81 \text { ó } \leq 15 \\
\geq 71 \text { ó } \leq 12 \\
\geq 51 \text { ó } \leq 10 \\
\geq 30 \text { ó } \leq 9\end{array}$ \\
\hline $\begin{array}{l}\text { Esfuerzo } \\
\text { respiratorio }\end{array}$ & & Normal & Aumento leve & $\begin{array}{l}\text { Aumento } \\
\text { moderado }\end{array}$ & $\begin{array}{c}\text { Severo incremento/ } \\
\text { apnea }\end{array}$ \\
\hline $\begin{array}{l}\text { Saturación de } \\
\text { oxígeno (\%) }\end{array}$ & & $>94$ & 91 a 94 & $\leq 90$ & \\
\hline Terapia de oxígeno & & Aire ambiente & & $<4$ L/min ó < 50\% & $\geq 4$ L/min ó $\geq 50 \%$ \\
\hline
\end{tabular}

se referían a los eventos adversos ocurridos durante el transporte de pacientes adultos, distinguiendo eventos adversos mayores y menores. Los eventos adversos mayores implican riesgo vital y actuación inmediata del equipo de transporte, estos son clasificados en: cardiocirculatorios, respiratorios, neurológicos, mal funcionamiento de equipo y errores humanos $^{16}$. Variadas publicaciones refieren que el riesgo de eventos adversos es mayor que los pacientes pediátricos, requiriendo estabilización previa y un equipo experto en el manejo del paciente transportado ${ }^{12,13}$.

El nuevo PEWS modificado de 7 variables (tres circulatorias y cuatro respiratorias), hace muy factible su uso rutinario en la práctica clínica, ya que puede ser medido objetivamente en niños despiertos y dormidos, además de no requerir exámenes de laboratorio. Posteriormente, en el año 2011, el mismo Dr. Parshuram y su equipo, publicó un estudio multicéntrico de caso y controles con un total de 2.074 pacientes pediátricos, que subdividió en 686 casos y 1.388 controles, el nuevo PEWS resulto una herramienta altamente sensible y especifica para predecir deterioro del paciente pediátrico con enfermedad aguda ${ }^{21}$.

Casi simultáneamente, una revisión sistemática del Dr. Chapman y cols, publicada el 2010, concluye que existe evidencia contundente para calificar el PEWS como una herramienta válida, fiable y útil para identificar pacientes pediátricos en riesgo de deterioro, con una sensibilidad del $85 \%$ para puntuaciones sobre $3^{22}$.

Finalmente, lo expuesto permite concluir que realizar la evaluación del PEWS al inicio y al final del transporte, puede ayudar a definir la necesidad del transporte y a la vez objetivar el deterioro fisiopatológico sufrido por el paciente pediátrico durante su traslado. Durante el año 2012 fue publicada una investigación retrospectiva, que estudió a niños transportados al centro de salud de Atlanta, concluyendo que el $60 \%$ recibió alguna intervención por el equipo de traslado. Dentro del estudio se utilizo la herramienta PEWS para evaluar gravedad del paciente al inicio y al final del tras- 
lado, resultando estadísticamente significativo un aumento del PEWS al final del trasporte, el estudio concluye que el uso del PEWS para el transporte de pacientes puede funcionar como una herramienta útil para evaluar severidad del paciente, optimizar el transporte y evaluar el deterioro fisiopatológico de este $22-24$.

\section{Referencias}

1.- Ministerio de Salud (MINSAL): [Internet] Misión y Visión 2011. [consultado 10 de junio de 2011]. Disponible en: http://www.minsal.gob.cl/portal/url/page/ $\mathrm{minsalcl} / \mathrm{g} \_$conozcanos/g_mision_vision/presentacion_mision_vision.html.

2.- Cortés P: División de Gestión Red Asistencial (DIGERA). Estadística SAMU 2013. [presentación no publicada].

3.- $\quad$ Stroud MH, Trautman MS, Meyer K, et al: Pediatric and neonatal interfacility transport: results from a national consensus conference. Pediatrics 2013; 132: 359-66.

4.- Ramnaryan P, Thiru K, Parslow RC, et al: Effect of specialist retrieval teams on outcomes in children admitted to paediatric intensive care units in England and Wales: a retrospective cohort study. Lancet 2010; 376: 698-704.

5.- Suominen P, Olkkola KT, Voipio V, Korpela R, Palo R, Räsänen $J$ : Informe estilo Utstein intrahospitalaria de reanimación cardiopulmonar pediátrica. Reanimación 2000; 45: 17-25.

6.- Reis AG, Nadkarni V, Perondi MB, Grisi S, Berg RA: Una investigación prospectiva en la epidemiología de la intrahospitalaria reanimación cardiopulmonar pediátrica utilizando el estilo internacional de informes Utstein. Pediatrics 2002; 109: 200-9.

7.- Tibballs J, S Kinney, Duke T, Oakley E, Hennessy M: Reducción de la pediatría en paciente con paro cardíaco y la muerte con un equipo de emergencia médica: resultados preliminares. Arch Dis Child 2005; 90: 1148-52.

8.- Stroud MH, Prodhan P, Moss M, Fiser R, Schexnayder $S$, Anand $K$ : Enhanced monitoring improves pediatric transport outcomes: a randomized controlled trial. Pediatrics 2011; 127 (1): 42-8.

9.- Petrillo-Albarano $T$ : The use of a modified pediatric early warning score to assess stability of pediatric patients during transport. Pediatr Emerg Care 2012; 28 (9): 878.

10.- Duncan H, Hutchison J, Parshuram CS: The Pediatric Early Warning System score: a severity of illness score to predict urgent medical need in hospitalized children. J Crit Care 2006; 21: 271-8.
11.- Parshuram CS, Hutchison J, Middaugh K: Development and initial validation of the Bedside Paediatric Early Warning System score. Crit Care Forum 2009; 13: 1-10.

12.- American Heart Association. Reanimación cerebral: Tratamiento del cerebro después de la reanimación cardíaca. En: Reanimación Cardiopulmonar avanzada. AHA 2010; 14: 1-5.

13.- Minimum standards for intrahospital transport of critically ill patients. Emerg Med (Fremantle) 2003; 15: 202-4.

14.- Gillman L, Leslie $G$, Williams T, Fawcett $K$, Bell R, McGibbon $V$ : Adverse events experienced while transferring the critically ill patient from the emergency department to the intensive care unit. Emerg Med J 2006; 23: 858-61.

15.- Stevenson VW, Haas $C F$, Wahl WL: Intrahospital transport of the adult mechanically ventilated patient. Respir Care Clin N Am 2002; 8: 1-35.

16.- Fanara B, Manzon C, Barbot O, Demestre T, Capellier $G$ : Recommendations for the intrahospital transport of critically ill patients. Critical Care 2010; 14: 2-10.

17.- Tice $P$ : Intrahospital transport of critically ill adults: potential physiologic changes and nursing implications. Focus Crit Care 1991; 18: 424-8.

18.- Thompson M, Van den Bruel A, Verbakel J, et al: Systematic review and validation of prediction rules for identifying children with serious infections in emergency departments and urgent-access primary care. Health Technol Assess 2012; 16: 1-100.

19.- Solevag A, Eggen E, Schroder J, Nakstad B: Use of a modified pediatric early warning score in a departamento of pediatric and adolecent medicine. Plos One 2013; 8: 210-7.

20.- Petrillo-Albarano T: The use of a modified pediatric early warning score to assess stability of pediatric patients during transport. Pediatr Emerg Care 2012; 28 (9): 878.

21.- Parshuram et al: Multicentre validation of the bedside paediatric early warning system score: a severity of illness score to detect evolving critical illness in hospitalised children. Critical Care 2011; 15: R184.

22.- Chapman SM, Grocott MP, Franck LS: Systematic review of paediatric alert criteria for identifying hospitalised children at risk of critical deterioration. Intensive Care Med 2010; 36: 600-11.

23.- Borrows EL: Effect of patient and team related factors on stabilization time during pediatric intensive care transport. Pediatr Crit Care Med 2010; 11 (4): 451-6.

24.- Skaletzky SM, Raszynski A, Totapally BR: Validation of a Modified Pediatric Early Warning System Score: A Retrospective Case-Control Study. Clin Pediatr 2012; 51 (5): 431-5. 